\title{
REHISTORIAR LA BIOÉTICA EN LATINOAMÉRICA LA CONTRIBUCIÓN DE JAMES DRANE
}

\section{Fernando Lolas Stepke*}

Resumen: Este artículo presenta la persona y la obra de James Drane en su relación con la bioética en Latinoamérica. Tras un breve examen de su carrera y publicaciones, se destaca la creación de un centro de estudios en Edinboro University of Pennsylvania, legado de la filantropía de Russell B. Roth y su familia, que perpetuará el espíritu de interrogación libre y lealtad a sus principios que Drane ha mantenido a lo largo de su vida.

Palabras clave: bioética en Latinoamérica, OPS, James Drane

\section{REMAKING BIOETHICS' HISTORY IN LATIN AMERICA. JAMES DRANE'S CONTRIBUTION?}

\begin{abstract}
This article presents the person and work of James Drane in their relation to bioethics in Latin America. After a brief analysis of his career and publications, the establishment of a study center at the Edinboro University of Pennsylvania is underscored, a legacy of the late Russell B. Roth and his family, which will perpetuate the spirit of free inquiry and loyalty to his principles that Drane has kept throughout his life.
\end{abstract}

Key words: Latin American bioethics, PAHO, James Drane

\section{RE-HISTORIAR A BIOÉTICA NA AMÉRICA LATINA. A CONTRIBUIÇÃO DE JAMES DRANE}

Resumo: Este artigo apresenta a pessoa e a obra de James Drane relacionada com a bioética na América Latina. Após um breve exame de sua carreira e publicações, destaca-se a criação de um centro de estudo na Edinboro Univerisity na Pensilvânia (EUA), legado da filantropia de Russell B. Roth e sua família. Este centro perpetuará o espírito de livre pensador e lealdade aos seus princípios que Drane tem defendido ao longo de sua vida.

Palavras chave: bioética na América Latina, OPAS, James Drane 


\section{El contexto}

Ahora que la palabra "bioética" está de moda, medran a su amparo y cobijo muchas personas, con derecho o sin él. Hay gurúes, expertos, aficionados, políticos, advenedizos, gerentes, en fin, una fauna diversa y abundante. Algunos más prolíficos que otros, estos distintos cultores de la bioética suelen repetir una historia fundacional uniforme porque la han leído en las fuentes más habituales. A veces, suelen acomodarla a sus particulares intereses.

La verdad es que en cualquier ámbito asociado con este término es difícil no obtener la impresión de que cada actor que entra al juego bioético, o dice participar de su discurso, abriga una concepción particular, tiene intenciones especiales y obtiene productos disímiles. En este caso, quizá más que en otros, debe tenerse presente la noción orteguiana del "punto de vista". Desarrollar este tema permitiría establecer cuánto diálogo hay entre diversos usuarios del término "bioética". Ello sería útil, además, porque necesitamos una mirada ética sobre lo que es hoy la bioética. La simple enunciación de buenos fines -la bioética los tiene- parece bastar para atribuir pureza de medios a quienes dicen cultivarla. Lo cual no siempre es el caso. La noción de una disciplina intelectual con sus correspondientes "sabios", "expertos" y "políticos" necesita ser clarificada en un campo tan difuso y tan lábil como el que la bioética ha demostrado ser.

No es tal examen designio del presente texto. Nuestras afirmaciones se hacen desde Iberoamérica y desde una posición especial -servidor público de instituciones de cultivo intelectual y servicio.

Una primera comprobación: la bioética tiene un largo pasado pero una breve historia. Mucho antes de que se difundiera el vocablo, las preocupaciones que identifica ya existían, como exis- tían también conflictos, soluciones y dilemas. Otro punto digno de mención: más o menos en la misma época (segunda mitad del siglo XX) la preocupación cristalizó en muchas iniciativas que podemos llamar "institucionalizantes": se fundaron institutos, se crearon grupos de trabajo, se editaron textos con el epígrafe "bioética". Esto indica que la palabra vino a ser una suerte de cristalización de algo que se necesitaba.

Pero la palabra no es la cosa. Sesudas explicaciones y alguna que otra tontería se basan en eso que Mainetti llama "la cuestión nominal". La trivial repetición de que es término compuesto de "bios" y "ethos" suele ocupar a muchos que creen con ello explicar algo. En realidad, se trata de un ejercicio etimológico que responde una pregunta poco relevante. Lo que necesitamos saber, en realidad, es qué hacen quienes dicen "hacer bioética", en qué invierten sus horas y días, qué esperan lograr, cuáles son sus motivaciones. Aquí hay una profusión de respuestas y una cantidad de hechos que poco tienen que ver con la etimología de este neologismo afortunado y vago.

Quien recibe el crédito mayor por inventar el neologismo no anticipó lo que vendría a ser la bioética y más bien deploró el sentido que adquirió el vocablo. En realidad, Van Rensselaer Potter quiso después hablar de "ética global". Su visión era la de un profeta de la catástrofe ecológica que amenazaba la supervivencia humana. Actualmente, el significado de bioética suele restringirse, en la mayoría de los casos, a una fusión de humanidades y medicina o ciencias de la salud, o a la toma de decisiones éticas en el contexto de instituciones sanitarias. Se encuentra un eco en los escritos de los científicos, a menudo para reclamar si una supervisión ética de sus trabajos coartará su creatividad. A veces tienen razón, porque bajo la rúbrica de bioética puede hacerse daño sin un adecuado trabajo de preparación. 
En lo que sigue, quiero rehistoriar la bioética evitando caer en los vicios que señalo, de los cuales también he sido culpable sin vergüenza ni disculpa. La "rehistoria" es una historia contada de nuevo, y es tarea de nunca acabar. Pruebo ahora con la perspectiva que me ha dado James -Jim- Drane en su lugar de trabajo, la Universidad de Edinboro de Pennsylvania. Este ejercicio de rehistoria parte, en este caso, del trabajo concreto de un individuo particular que contribuyó a mucho de lo que hoy conocemos bajo bioética en América Latina.

\section{La bioética en América Latina y James Drane}

En mis viajes, conversaciones y lecturas me he topado con muchos pioneros. Sabios vaticinadores del porvenir, a menudo incomprendidos, que lamentan el poco eco de sus profecías o milagrosas elucubraciones. Siempre miro con simpatía tales declaraciones. Me recuerdan que la razón siempre acompaña a los vencedores, a quienes tienen éxito. Me hacen pensar que la popularidad no es lo mismo que el prestigio y que, si hubiera justicia en el mundo, la victoria llegaría a todos ellos. Además, habida cuenta de la aparente buena voluntad de algunas personas, suelo recordar que Ludwig Klages advertía que raramente una gran alma coexiste con un gran espíritu. Las luces intelectuales no son garantía de bondad moral. Pero ésta tampoco lo es de pensamiento riguroso. La confusión proviene, creo, de que la voz "ética" viene a ser empleada en un sentido sentimental y dulzón, como si al usarla pudiéramos prescindir de una sana controversia y de dura polémica intelectual.

Estas consideraciones debieran anteceder toda exposición histórica sobre instituciones y personas en este ámbito. Juzgará el lector, cuando ella concluya, si son acertadas y si, lo que importa, ayudan a discernir y valorar.
No sé exactamente cómo conocí a James Drane, Jim para todo el mundo. Solamente recuerdo que un día ya lejano se presentó en mi oficina de la Universidad de Chile para hablarme de un proyecto que tenía entre manos. Pudo ser 1988 ó 1989. Era verano y yo aún estaba en el Departamento de Fisiología y Biofísica de la Facultad de Medicina. Ese día, que culminó con un almuerzo en el restaurante "Omar Khayyam" (después supe que Jim no podía siquiera probar la carne, por el colesterol), confirmé que la Organización Panamericana de la Salud (OPS) planeaba establecer un programa regional de bioética. Mi nombre estaba entre las personas que Jim, como consultor itinerante, debía contactar para ganar una impresión de primera mano sobre la ética médica y las profesiones de la salud.

El proyecto consistía en instalar en OPS un organismo técnico que supervisara éticamente los trabajos de la organización. Básicamente, se había sentido la carencia de lineamientos apropiados en materia de investigación biomédica y de provisión de servicios. Aunque problema básicamente jurídico, tenía aristas éticas que era necesario abordar. Por eso, la bioética en OPS nació asociada a la oficina jurídica y por eso, también, tuvo el abogado de la organización un papel en su gestación y desarrollo.

Con Jim y otras personas mantuvimos contacto epistolar y la primera muestra de esos trabajos fue el volumen especial del Boletín de la Oficina Sanitaria Panamericana dedicado al tema, el cual sería republicado luego como libro, bajo el título "Bioética: temas y perspectivas", tanto en inglés como en español(1).

En otro sitio he mencionado ya que la palabra y sus connotaciones (las de entonces, no las actuales) me habían llegado en 1986 a través de José Alberto Mainetti, y se habían fortalecido en sus tradicionales coloquios novem- 
brinos de La Plata, Argentina. Ese cenáculo fue uno de los crisoles en que se forjó la bioética latinoamericana. No el único, pero uno importante. Del magma germinal de lo que entonces conocíamos como humanidades médicas emergió el tema bioético de forma principal(2).

En esos años finales de la década de los ochenta, en que mis propios trabajos y estudios me orientaban hacia una mayor interpenetración disciplinaria, conocer a Mainetti, Diego Gracia, Hans-Martin Sass, Manuel Velasco-Suárez, Alfonso Llano, Roberto Llanos y otras personas que luego desempeñarían papeles en la bioética latinoamericana, fue importante. Así, al ser nombrado vicerrector de Asuntos Académicos y Estudiantiles en la Universidad de Chile, acompañando al rector Jaime Lavados en su gestión entre 1993 y 1998 , ya habíamos avanzado en formar una comisión primero y un centro de estudios después. Íntimamente relacionadas con el trabajo de nuestra oficina en Washington DC, liderada por Jorge Litvak, tales iniciativas desembocaron en una alianza con la Organización Panamericana de la Salud (OPS) y el establecimiento del Programa Regional de Bioética(3).

Que el Programa se estableciera en Santiago de Chile obedeció a muchos factores: el interés del jefe de la oficina jurídica de OPS, Hernán Fuenzalida, el trabajo que habíamos realizado en la Universidad de Chile y la recomendación, entre otros, de James Drane. OPS había encargado a Jim viajar por el continente. Escribió sendos informes sobre ese viaje y, tras algunos altibajos, se pudo concretar el acuerdo entre OPS, el Gobierno de Chile y la Universidad de Chile en enero de 1994. El Programa Regional se inició bajo la dirección de Julio Montt, ex ministro de Salud chileno.

Cuando, en 1998, dejé el cargo de vicerrector de la Universidad de Chile, el director de OPS, Dr. George Alleyne, me ofreció la dirección del Programa Regional. Desde entonces, la colaboración armoniosa entre el Gobierno chileno, la Universidad de Chile y el Ministerio de Relaciones Exteriores de Chile ha producido los frutos que originalmente se esperaron. Al cumplirse diez años de la instalación del Programa, ya convertido en Unidad de Bioética, recordamos sus comienzos, repasamos sus lineamientos generales y formulamos sus desafíos inmediatos. En esa conmemoración, como merecía, rendimos un homenaje a Jim Drane por sus aportes y su permanente disposición a ayudar(3).

\section{Una carrera particular}

Nacido en 1930, Jim Drane estudió en el Little Rock College, Arkansas, en la Universidad Gregoriana de Roma, en el Middlebury College, Estados Unidos, y en la Universidad de Madrid, España. Obtuvo grados en Teología, Lenguas Romances y Filosofía. Fue ordenado sacerdote en 1956. Después de estos estudios, realizó otros en Georgetown University, Yale University, Menninger Clinic y otras instituciones, ampliando sus experiencias y conocimientos en el campo de la medicina, la psiquiatría y las lenguas románicas.

Hacia 1967, James Drane enseñaba Filosofía y Lenguas en el Seminario de Little Rock, Arkansas. Ya era un sacerdote de promisoria carrera en el seno de la Iglesia, doctor en Filosofía por la Universidad de Madrid y admirado por su conocimiento de las Lenguas Romances. Había escrito una tesis sobre la libertad religiosa y la tolerancia. Era ya viajero impenitente, que había visitado secretamente la Unión Soviética y escrito un libro sobre su viaje ("Pilgrimage to Utopia"), el cual se tradujo al español y obtuvo buena resonancia. Al fin, hombre de pensamiento y reflexión, al cual los aires renovadores del Concilio Vaticano II hacían concebir grandes esperanzas. 
Las esperanzas de Jim, como de otros católicos en aquel tiempo, se cifraban en una renovación de la Iglesia Católica, no en su núcleo doctrinal pero sí en las prácticas concretas y el comportamiento de los creyentes. Los laicos querían un aggiornamento que permitiera, si no flexibilizar, al menos humanizar la ortodoxia. Entre los muchos temas que ocupaban por aquel tiempo las conciencias, el de la reproducción humana ocupaba un lugar importante. Eran los años posteriores a la introducción de la anticoncepción química, que liberaba a las mujeres de la obligada asociación entre placer del sexo y procreación. Eran años de cambio en las jerarquías, las cuales se remozarían si la autoridad brindaba ocasión renovada para el diálogo y la participación.

James Drane recordaba con dolorosa nitidez una entrevista que sostuvo al comienzo de su carrera sacerdotal con un joven matrimonio que, agotado por las deudas y las estrecheces económicas, no deseaba tener más hijos. La Iglesia Católica no les permitía más opciones que los llamados "métodos naturales" (como el Billings) para el control de una natalidad ya no deseada. De resultas de no poder ayudar a esa pareja, el padre Drane investigó durante años el tema y se formó una opinión, vertida en sendos artículos que publicó en el diario "Arkansas Gazette" en 1967. La resonancia de esos textos sobrepasó el ámbito de un periódico local. Cuando sólo faltaba una semana para que se terminara de publicar la serie, el padre Drane recibió una carta de su obispo indicándole que sus opiniones transgredían severamente la ortodoxia y que debía dejar sus tareas en el seminario.

Lo que siguió fue un proceso relativamente rápido, en el marco del derecho canónico, durante el cual James Drane reclamó su derecho a ser escuchado, apeló las decisiones de los tribunales, llegó hasta Roma. Se le ofreció, como alternativa, ser destinado a una lejana parro- quia y enmudecer para siempre, o retractarse públicamente de lo que había escrito. Jim no hizo ninguna de las dos cosas y solicitó ser destinado al estado laico. La descripción que hace de su última conversación con autoridades eclesiásticas, en Washington DC, cabría perfectamente en una antología de los desencuentros. Mientras se dirigía a la nunciatura apostólica, el chofer del taxi que lo llevaba le preguntó qué iba a hacer en esa embajada vaticana. Al responder Jim, el taxista detuvo su vehículo y, casi como la voz innominada del pueblo llano, lo conminó a persistir en sus convicciones, a defender la coherencia de su pensamiento y a brindar, con su ejemplo, una esperanza para la Iglesia y su renovación.

En seguida inició una búsqueda de trabajo, primero en la Universidad de Yale, donde le acogió el teólogo protestante Gustafson, y luego en lo que más tarde sería la Universidad de Edinboro de Pennsylvania, en la cual se quedó enseñando y trabajando hasta jubilar. Su llegada a esa universidad fue un azar climático. Viajando en avión, una tormenta de nieve obligó a Jim a quedarse varios días en esa localidad cercana al lago Erie. Le dio tiempo para conversar con las autoridades, conocer el lugar, apreciar las bondades del retiro provinciano y decidir quedarse.

A lo largo de su vida profesional, Jim Drane ha mantenido amistades duraderas con personas de muy diversa persuasión, pero se mantuvo fiel a la religión católica y sus ritos. En la época germinal del Hastings Center compartió proyectos e iniciativas con Daniel Callahan y conoció a todos los iniciadores y gestores del Kennedy Center de la Universidad de Georgetown, además de a casi la totalidad de los académicos y estudiosos que contribuyeron a delinear el campo de lo que después sería la bioética. Para mí, en lo personal, Drane y sus consejos han significado proyectar sobre mis iniciativas y trabajos el aura de una sol- 
vencia intelectual y una apertura de horizontes que han satisfecho muchos de mis intereses. Una de sus primeras acciones fue hacer que me invitaran a una importante reunión del Park Ridge Center de Chicago, en 1990, durante la cual no solamente conocí a muchos destacados cultores de este novel campo de estudios, sino también tuve una visión de primera mano sobre las "nuevas voces" y las nuevas orientaciones en la bioética de entonces.

\section{Las contribuciones de James Drane}

No es el propósito de este texto evaluar críticamente los libros y artículos de Drane. Su pensamiento ha escudriñado aspectos muy diversos de la realidad social y se ha enfrentado a entornos culturales y académicos muy heterogéneos. Su activa participación como conferenciante en congresos, seminarios y talleres le ha convertido en un invitado bienvenido en muchos países y su fluido dominio de la lengua española ha garantizado su presencia en prácticamente todos los países hispanoparlantes en donde se ha desarrollado la preocupación bioética.

Después de escribir su todavía inédita tesis sobre la tolerancia y la libertad religiosa (premonitoria elección de tema, considerando las peripecias ulteriores), Drane publicó "Authority and institution", libro que en 1969, dos años después de su expulsión de las filas del clero, quería sugerir que la estructura de las instituciones religiosas no es parte de la verdad revelada, sino un instrumento que debe entenderse en el contexto político que es propio de sus gestores y con fundamento en sus raíces históricas.

A ese libro siguió "A new american reformation" en 1974, "Religión and ethics" en 1977 y "Your emotional life" en 1984. Estos escritos tienen en común el intento por comprender la circunstancia histórica y personal de la nueva juventud de esos años, los avatares biográficos del autor y lo que había obtenido de su paso por la famosa Menninger Clinic y haber tomado contacto con la psiquiatría y las ciencias del comportamiento.

En "Becoming a good doctor", de 1989, se reconoce la impronta de Pedro Laín Entralgo y José Luis Aranguren, ambos amigos y maestros de Drane. Este libro recuerda que la mayor parte de los ejemplos usados en los cursos de Teología Moral proviene de la Medicina. También destaca que ésta es un campo privilegiado para el estudio de cuestiones éticas y que cualquier reflexión sobre la virtud estará bien servida si considera lo que acontece en el ámbito privilegiado de esta profesión. El libro tuvo una edición española en 1993, publicada en Bogotá, Colombia.

“Clinical Bioethics", publicado en 1994, fue concebido como un texto práctico que ayudara a los comités de ética en la toma de decisiones. Es un libro eminentemente útil, orientado al lector atareado, que precisa una rápida orientación sin perder rigurosidad.

En 1997, Drane publicó el libro "Caring to the end", otro trabajo de corte eminentemente práctico, que enseña ética a quienes laboran en salas de cuidado intensivo o en el marco de instituciones de atención de enfermos terminales. En 1999, la Organización Panamericana de la Salud publicó la traducción que preparamos en Santiago de Chile con el título El cuidado del enfermo terminal. Se trata de un texto que ha tenido una buena acogida entre los profesionales de la salud(4).

El libro "More humane medicine", de 2003, inauguró la flamante Edinboro University Press y fue traducido al portugués en conjunto con Leo Pessini. Su traducción al español ha quedado demorada, probablemente por la necesidad de reconsiderar algunos puntos polémicos 
que este texto destaca. Baste señalar que su subtítulo reza "A liberal Catholic bioethics", lo cual puede ser complejo de aceptar en algunos círculos.

A los libros mencionados deben agregarse los numerosos artículos publicados por Drane en distintos medios técnicos y de prensa. Algunos de ellos han sido extensamente usados como material de enseñanza en cursos dictados en diferentes instituciones.

\section{Balance y perspectivas: un aporte perdurable}

$\mathrm{Si}$ alguien me pidiera resumir lo que sé de James Drane diría que es un scholar que no ha transado los principios de la fe de su infancia pero ha intentado reformularlos en un contexto de tolerancia y apertura. Ha tenido la dicha de una carrera académica en un oasis como la universidad que lo ha acogido, ejemplo característico de esa combinación de provinciana vida bucólica y demandas docentes e intelectuales que son los centros de estudio del tipo de Edinboro University of Pennsylvania.

Al retirarse de la vida activa, Jim ha querido testimoniar su compromiso perdurable con la causa bioética en el continente latinoamericano y caribeño, legando los recursos que pu- siera a disposición de la universidad el difunto Russell B. Roth, presidente que fue de la American Medical Association, amigo y mecenas. El establecer un Centro de Estudios que llevará su nombre permitirá ofrecer a los cultores de la bioética un refugio intelectual libre de imposiciones doctrinarias y será un estímulo para la producción intelectual. Para quien escribe estas líneas, haber sido nominado como el primer scholar de este naciente centro en 2005 y contribuir a gestar la arquitectura de su gestión, constituyen dos motivos de tranquila satisfacción y orgullo.

Cuando deba escribirse la historia real de la bioética en nuestro continente, sin duda algunas de las ideas expresadas en este trozo de rehistoria serán parte del relato oficial. Para cuando eso ocurra, habrá desaparecido, espero, esa efervescencia de los comienzos y el vocinglero tercermundismo militante que algunos confunden con la lucha por la equidad de los pueblos. Habremos dejado atrás, imagino, la villanía intelectual que lleva a protagonismos mal inspirados y negativos. Se habrá adquirido la respetabilidad de un campo de estudio sobrio y productivo, acorde con la circunstancia en que se desarrolla, fiel a sus raíces y muestra de excelencia. Cuando eso ocurra, la figura de James Drane deberá alinearse junto a las más importantes en esta empresa.

\section{Referencias}

1. OPS Bioética. Temas y perspectivas. Washington DC: Organización Panamericana de la Salud; 1990 (hay edición en inglés del mismo año). Publ. Científica $\mathrm{N}^{0} 527$.

2. Lolas F. Bioética y antropología médica. Santiago de Chile: Editorial Mediterráneo; 2000.

3. Lolas F, (ed.) Diálogo y cooperación en salud. Diez años de bioética en la OPS. Santiago de Chile: Unidad de Bioética, Organización Panamericana de la Salud OPS/OMS; 2004.

4. Drane JF. El cuidado del enfermo terminal. Washington, DC: Organización Panamericana de la Salud; 1999. 